\title{
Performance of a fully-automated system on a WHO malaria microscopy evaluation slide set
}

\author{
Matthew P. Horning ${ }^{1 *}$ (D) Charles B. Delahunt ${ }^{1,2}$, Christine M. Bachman ${ }^{1}$, Jennifer Luchavez ${ }^{4}$, Christian Luna ${ }^{4}$, \\ Liming Hu', Mayoore S. Jaiswal ${ }^{3}$, Clay M. Thompson ${ }^{5}$, Sourabh Kulhare', Samantha Janko ${ }^{6}$, Benjamin K. Wilson', \\ Travis Ostbye ${ }^{1}$, Martha Mehanian ${ }^{1}$, Roman Gebrehiwot ${ }^{3}$, Grace Yun ${ }^{3}$, David Bell ${ }^{7}$, Stephane Proux ${ }^{8}$, \\ Jane Y. Carter ${ }^{9}$, Wellington Oyibo ${ }^{10}$, Dionicia Gamboa ${ }^{11}$, Mehul Dhorda ${ }^{12}$, Ranitha Vongpromek ${ }^{13}$, \\ Peter L. Chiodini ${ }^{14}$, Bernhards Ogutu ${ }^{15}$, Earl G. Long ${ }^{16}$, Kyaw Tun ${ }^{17}$, Thomas R. Burkot $^{18}$, Ken Lilley ${ }^{19}$ \\ and Courosh Mehanian ${ }^{1}$
}

\begin{abstract}
Background: Manual microscopy remains a widely-used tool for malaria diagnosis and clinical studies, but it has inconsistent quality in the field due to variability in training and field practices. Automated diagnostic systems based on machine learning hold promise to improve quality and reproducibility of field microscopy. The World Health Organization (WHO) has designed a 55-slide set (WHO 55) for their External Competence Assessment of Malaria Microscopists (ECAMM) programme, which can also serve as a valuable benchmark for automated systems. The performance of a fully-automated malaria diagnostic system, EasyScan GO, on a WHO 55 slide set was evaluated.

Methods: The WHO 55 slide set is designed to evaluate microscopist competence in three areas of malaria diagnosis using Giemsa-stained blood films, focused on crucial field needs: malaria parasite detection, malaria parasite species identification (ID), and malaria parasite quantitation. The EasyScan GO is a fully-automated system that combines scanning of Giemsa-stained blood films with assessment algorithms to deliver malaria diagnoses. This system was tested on a WHO 55 slide set.

Results: The EasyScan GO achieved $94.3 \%$ detection accuracy, $82.9 \%$ species ID accuracy, and $50 \%$ quantitation accuracy, corresponding to WHO microscopy competence Levels 1, 2, and 1, respectively. This is, to our knowledge, the best performance of a fully-automated system on a WHO 55 set.

Conclusions: EasyScan GO's expert ratings in detection and quantitation on the WHO 55 slide set point towards its potential value in drug efficacy use-cases, as well as in some case management situations with less stringent species ID needs. Improved runtime may enable use in general case management settings.
\end{abstract}

Keywords: Malaria, Automated diagnosis, Machine learning, Microscopy, WHO

*Correspondence: matthew.horning@ghlabs.org

${ }^{1}$ Global Health Labs (formerly at Intellectual Ventures Laboratory/Global Good), 14360 SE Eastgate Way, Bellevue, WA 98007, USA

Full list of author information is available at the end of the article

\section{Background}

Microscopic examination of Giemsa-stained blood films continues to be widely used for parasitological confirmation of malaria diagnosis, representing roughly half of all malaria diagnostic tests performed worldwide in 2018 [1]. Assessments of the therapeutic efficacy

c) The Author(s) 2021. This article is licensed under a Creative Commons Attribution 4.0 International License, which permits use, sharing, adaptation, distribution and reproduction in any medium or format, as long as you give appropriate credit to the original author(s) and the source, provide a link to the Creative Commons licence, and indicate if changes were made. The images or other third party material in this article are included in the article's Creative Commons licence, unless indicated otherwise in a credit line to the material. If material is not included in the article's Creative Commons licence and your intended use is not permitted by statutory regulation or exceeds the permitted use, you will need to obtain permission directly from the copyright holder. To view a copy of this licence, visit http://creativeco mmons.org/licenses/by/4.0/. The Creative Commons Public Domain Dedication waiver (http://creativecommons.org/publicdomain/ zero/1.0/) applies to the data made available in this article, unless otherwise stated in a credit line to the data. 
of anti-malarial drugs depend on microscopy for the detection and identification of malaria parasites and for estimation of parasite density. However, difficulties in maintaining consistent access to training, quality assurance, and material resources can lead to high variability in the quality of microscopy, hindering programmatic and research operations in the malaria endemic regions where they are most needed [2,3].

Malaria microscopy is thus a high-value target for automated image-processing and machine learning (ML) systems because such systems can potentially be widely deployed, mitigating the expert-training bottleneck, and because their results are reproducible. Since a thorough review in 2018 [4], there have been several additional reports proposing or evaluating systems for automated interpretation of malaria blood films [5-14].

A lack of benchmark datasets hampers the evaluation and comparison of various automated systems $[4,5]$. One strong candidate for such a benchmark is the slide set used for the World Health Organization (WHO) External Competence Assessment of Malaria Microscopists (ECAMM) programme. These slide sets consist of 55-56 carefully specified Giemsa-stained blood films, used to evaluate microscopists in detection, species ID, and quantitation of malaria parasites as part of the WHO Quality Assurance programmes $[15,16]$. Assessment based on an ECAMM slide set can offer valuable insight into a system's effectiveness, because it focuses on the key requirements for viability in field use-cases. Crucially, a system that hopes to deploy through existing field infrastructure, clinicians, and protocols needs to operate successfully on standard Giemsa-stained blood films as currently prepared and processed in the field by microscopists.

This paper reports performance of a fully-automated end-to-end malaria diagnostic system, the Motic EasyScan GO [17], on an ECAMM slide set. The system includes (i) an automated bright-field microscopy platform for scanning of Giemsa-stained thick and thin blood films, and (ii) malaria detection algorithms, previously described in $[5,6]$, that process the image sets to give parasite detection, species ID, and parasite quantitation at the patient level.

\section{Methods}

\section{ECAMM test set}

A set of Giemsa-stained blood films consisting of both malaria positive and negative cases and varying parasite densities were obtained from the WHO WPRO (Regional Office for the Western Pacific) regional malaria slide bank, a collection of reference slides managed by the WHO Collaborating Centre for malaria diagnosis at the Research Institute for Tropical Medicine (RITM) [18].
All slides in the slide bank were previously validated by 12 independent microscopists certified as Level 1 malaria microscopists through the WHO ECAMM. Each microscopist read two representative slides from a set of about 200 slides per case. The parasite species was confirmed by at least $70 \%$ of the readers and by polymerase chain reaction (PCR). Parasite counts per microliter were estimated against 500 white blood cells and calculated with an estimated average white cell count of $8000 / \mu \mathrm{L}$, and the median of 24 readings was taken as the reference count. Slides with statistically significant inter- or intra-reader count variation are never selected for the ECAMM sets. The 55-slide set used in the experiments for this study was assembled by WHO WPRO to represent the template for ECAMM slide sets described in [16] and shown in Fig. 1 (henceforth the WHO 55). However, it had a few deviations. Such deviations are common in ECAMM workshops (K. Lilley, pers. commun.) due to the logistical difficulties of assembling an ideal set. The deviations present in this set are described below, and their effects on the results are discussed in the Discussion section. For detailed contents of the set used in this study, see Additional File 1.

The ECAMM slide set is designed to evaluate a microscopist's competence in three key areas important for malaria diagnosis: (1) malaria parasite detection, (2) malaria parasite species ID, and (3) quantitation of malaria parasites. Accuracy in each of these areas is evaluated using a different subset of the ECAMM slide set (see [16] and Fig. 1):

\section{Detection}

Ideally, the detection component uses 20 negative samples and 20 positive samples with a parasitaemia ranging from 80 to 200 parasites per microlitre $(\mathrm{p} / \mu \mathrm{L})$. Given the samples in the set provided, the Plasmodium falciparum samples with a parasitaemia below $200 \mathrm{p} / \mu \mathrm{L}$ (7 slides, rather than 10) and all non-falciparum and mixed-species samples (8 slides rather than 10) were used to evaluate detection. The non-falciparum slides ranged from 164 to $10,184 \mathrm{p} / \mu \mathrm{L}$ with most samples above $1000 \mathrm{p} / \mu \mathrm{L}$, well above the ideal range of $80-200 \mathrm{p} / \mu \mathrm{L}$.

\section{Species identification}

Ideally, the species ID component uses the same samples, 20 negative and 20 positive, as the detection subset. Negative samples are used along with positive samples for evaluating species ID accuracy during ECAMM (K. Lilley, pers. commun.). The same subset of slides, 20 negative and 15 positive, used for Detection were used for Species ID. The provided set also contained only one mixed-species sample, rather than four as called for by 


\title{
BOX 1 Model Minimum Slide Sets for Accreditation of Trained Laboratory Staff in Malaria Microscopy at National Reference (Core) Group Level.
}

\author{
Slide set 1 (40 slides): Assessment of presence/absence of parasites, and species \\ identification \\ - 20 negative slides: \\ -20 'clean' negatives \\ - 20 positive slides of low density (80-200 parasites/ $\mathrm{LL}$ ): \\ - 10 Plasmodium falciparum slides \\ - 4 mixed (2) species slides (Include P. falciparum. Each species $>40$ parasites $/ \mu \mathrm{L}, \mathrm{co}-$ \\ infecting species according to local prevalence) \\ - 6 of Plasmodium malariae, Plasmodium vivax, and/or Plasmodium ovale slides (include at \\ least 1 of each species, ratio according to local prevalence) \\ Time limit: 10 minutes per slide \\ Slide set 2 (15 positive slides): Assessment of quantitation \\ - 3-5 P. falciparum (200-500 parasites/ $\mu \mathrm{L}$, \\ - 9-10 P. falciparum (500-2000) \\ - 2 P. falciparum >100 000 parasites $/ \mu \mathrm{L}$ ) \\ Time limit: 10 minutes per slide
}

Fig. 1 Contents of ECAMM evaluation slide set. From [16]

the WHO malaria microscopy quality assurance manual [16].

\section{Quantitation}

Ideally, the quantitation component uses 15 P. falciparum slides with parasitaemias within $200 \leq \mathrm{p} / \mu \mathrm{L} \leq 2000$, plus one or two very high parasitaemia slides. From the provided set, the 20 P. falciparum slides with parasitaemia $>200 \mathrm{p} / \mu \mathrm{L}$ (rather than 15) were used. Of these, 18 slides had $200 \leq \mathrm{p} / \mu \mathrm{L} \leq 2000$, and two slides had $\sim 200,000 \mathrm{p} / \mu \mathrm{L}$. An estimated parasitaemia within $25 \%$ of the reference value was considered correct, per the WHO malaria microscopy quality assurance manual [16]. The parasitaemia range is important, because slides with low parasitaemias have higher Poisson variability and are thus harder to quantitate accurately (as provided in the supplementary information of reference [19]).

A newer, slightly modified version ("V2") of the ECAMM slide set template, with detailed protocols on its use during evaluation of microscopists, is found in [15]. "V1" [16] was used in this study, because (i) the provided ECAMM set was designed to match "V1"; and (ii) the "V2" protocols describe using a subset of the slides during an orientation phase prior to testing, which does not align with standard machine learning practice (the samples used to test an algorithm should never be used during training).

\section{Slides used for algorithm development}

The malaria detector algorithm was trained and validated using over 500 slides from 11 countries. Sources of training data (with geographic source of the slides in parentheses) included Shoklo Malaria Research Unit (Thailand), Amref Health Africa (Kenya), University of Lagos (Nigeria), Universidad Peruana Cayetano Heredia (Peru), World Wide Antimalarial Resistance Network (Thailand, Indonesia, Cambodia, and DR Congo), Hospital for Tropical Diseases and the London School of Hygiene and Tropical Medicine (UK), Kenya Medical Research Institute (Kenya), Centers for Disease Control and Prevention (USA and other countries), James Cook University (Solomon Islands), and the Defence Services Medical Academy (Myanmar).

No slides from RITM were used for training the machine learning algorithm models. A separate set of 48 slides from RITM, also roughly analogous to an official ECAMM set, was used along with the other slides listed above to tune final diagnostic parameters in the patient-level disposition step, prior to any exposure to the ECAMM test set. 


\section{Imaging method}

The slides, permanently mounted with cover glass, were scanned with a prototype version of the Motic EasyScan GO [17]. The microscope had a $40 \times, \mathrm{NA}=0.75$ objective, infinity-corrected optical train, and $10 \mathrm{~W}$ LED Köhler illumination. A CMOS camera captured images $(2048 \times 1536$ pixels $)$ at an approximate pixel pitch of 8.3 pixels $/ \mu \mathrm{m}$ at the sample plane. Each fieldof-view (FoV) was captured as a stack of 5 slices with $0.5 \mu \mathrm{m}$ vertical spacing, to ensure in-focus thumbnails of all objects-of-interest (e.g. parasites), which can lie at different depths due to the thickness of the blood films and/or slight tilting of the microscope slide.

On the thick film, a $20 \times 20$ grid of FoVs near the centre was manually selected and scanned. This gave an area scanned of roughly $18 \mathrm{~mm}^{2}$, equivalent to $\sim 580$ fields of view on a manual microscope with $100 \times$ oil immersion lens and $20 \mathrm{~mm}$ field number eyepiece, yielding $\sim 3 \mathrm{k}$ to $17 \mathrm{k}$ white blood cells (WBCs).

On the thin film, a rectangular region near the "feathered edge", where a monolayer of red blood cells (RBCs) is most often found, was manually selected and scanned, yielding 150 to $192 \mathrm{FoVs}$ and $\sim 10 \mathrm{k}$ to $50 \mathrm{k}$ non-overlapped RBCs. The area selected was based only on the presence of a monolayer of RBCs and not on whether parasites were visible in the region.

\section{Algorithm}

The image sets were analysed by both thick film and thin film malaria detector algorithms that detect and evaluate objects of interest. A final synthesis block combined the thick and thin film findings to produce a diagnosis report indicating: (i) whether malaria parasites were detected; (ii) the suspected malaria species; and (iii) the total parasite count (also ring-stage and late-stage counts) given as parasites per 8000 WBCs. This quantitation unit allows direct comparison to standard methods that assume a WBC density of $8000 / \mu \mathrm{L}$, but also allows for correction when the WBC count is known.

To assess performance on the ECAMM set, the results of the algorithm on each slide were compared with the reference readings from RITM, and performance was evaluated according to the rubric in the WHO malaria microscopy quality assurance manual [16] (shown in Table 1).

A schematic representation of the algorithm modules is shown in Fig. 2. Full algorithm details are found in [6] for thick film, and [5] for thin film. This section (i) provides a brief overview of the two algorithms; (ii) notes the updates to the current thick film algorithm from the version in [6]; and (iii) describes the synthesis block that
Table 1 Scoring rubric for ECAMM evaluation slide set, from [16]

\begin{tabular}{llll}
\hline $\begin{array}{l}\text { Accreditation } \\
\text { level }\end{array}$ & Detection & $\begin{array}{l}\text { Species } \\
\text { identification }\end{array}$ & $\begin{array}{l}\text { Parasite } \\
\text { quantitation }\end{array}$ \\
\hline $\begin{array}{l}\text { Based on lowest grade } \\
\text { achieved }\end{array}$ & $\begin{array}{c}\text { (within 25\% of } \\
\text { reference count) }\end{array}$ \\
1 & $\geq 90 \%$ & $\geq 90 \%$ & $\geq 50 \%$ \\
2 & $80 \%-<90 \%$ & $80 \%--<90 \%$ & $40 \%-<50 \%$ \\
3 & $70 \%--<80 \%$ & $70 \%--<80 \%$ & $30 \%-<40 \%$ \\
4 & $<70 \%$ & $<70 \%$ & $<30 \%$ \\
\hline
\end{tabular}

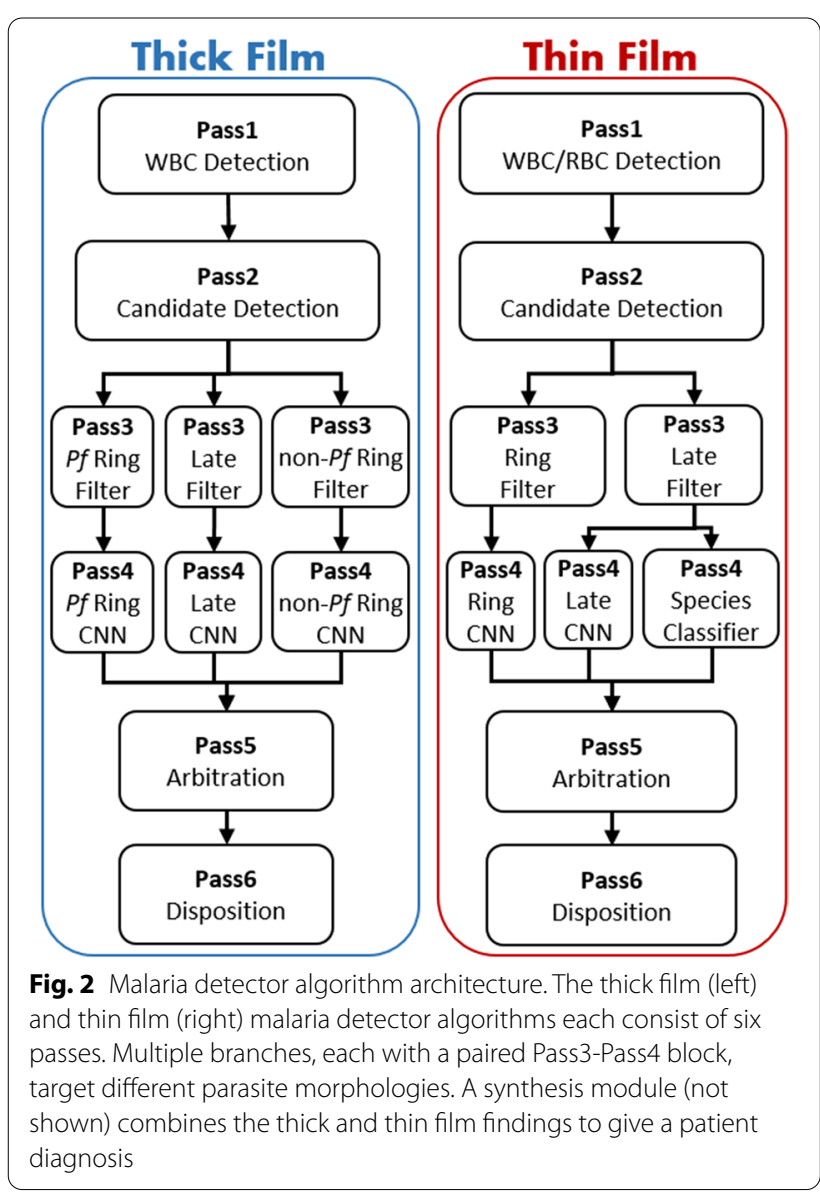

combines thick and thin film findings to give the final slide diagnosis.

\section{Algorithm overview}

Both algorithms consist of various processing blocks as depicted in Fig. 2. The term "Pass" refers to the different modules of processing (vertically laid out in Fig. 2) from Pass1 at the top to Pass6 at the bottom. The term "branch" refers to multiple blocks in Pass3, Pass4 
processing ( $P f$ Ring, Late, non- $P f$ Ring, horizontally laid out in Fig. 2). The processing performed in Pass1 through Pass6 is described below.

Pass1 Analyses every FoV stack for quality and focus. WBCs and RBCs are detected at the best focus level for each FoV. Thin film RBC and WBC detection use traditional computer vision techniques (e.g. thresholding, segmentation, feature extraction, classification), whereas the thick film uses a region-based convolutional neural network (CNN), Faster R-CNN [20], for WBC detection.

Pass2 Finds candidate parasites in all FoVs at all focus levels, via traditional computer vision techniques. The most in-focus thumbnail of each candidate object is selected for further processing.

Pass3 Weeds out obvious non-parasites (distractors) via traditional feature extraction and a gradient-boosted decision tree classifier [21].

Pass4 Applies a CNN classifier to thumbnails of all candidate parasite objects that survived the distractor filter of Pass3. In thin film only, a species classifier is applied to late-stage objects in a species branch [5].

Pass5 Arbitrates the appropriate stage type (ring or late) of objects detected by multiple branches, using the highest $\mathrm{CNN}$ score.

Pass6 (Disposition) processes all the detected and classified objects to (i) determine whether the blood film (thick or thin) is overall positive for malaria; (ii) estimate the parasitemia if positive; and (iii) determine the species of malaria if positive (potentially more than one).

Branches Multiple branches of paired Pass3-Pass4 modules are applied to the detected candidate objects. Each branch is trained and tuned to detect a different life-cycle stage of the malaria parasite. The ring-stage branch targets young ring-form trophozoites (in thick film only, there are separate $P$. falciparum and non-falciparum ring-stage branches). The late-stage branch targets latestage trophozoites, schizonts, and gametocytes, which are common in non-falciparum species and much rarer in $P$. falciparum. The species branch (thin film only) extracts a set of manually designed features for species ID, then classifies objects by species and stage via a gradient-boosted decision tree classifier.

\section{Thick film algorithm updates}

The thin film algorithm used in this study is identical to the one reported in [5]. The thick film algorithm is largely the same as the one reported in [6], but has been further developed:

(i) The algorithm in [6] was trained on images acquired with a prototype scanning microscope, whereas the results reported here are based on images scanned with the EasyScan GO [17].

(ii) The WBC detector is now based on a $\mathrm{CNN}$ object detection architecture, Faster R-CNN [20], as opposed to traditional computer vision techniques.

(iii) The distractor filter of Pass3 has been enhanced by doubling the number of discriminative features.

(iv) The thick-film algorithm has a new third detection branch targeting non-falciparum ring-stage trophozoites. This helps distinguish $P$. falciparum from non-falciparum infections solely from the thick film, even if a non-falciparum sample presents few or no late-stages, by recognizing that beyond the youngest ring-stages, non-falciparum and $P$. falciparum trophozoites differ morphologically.

(v) The Pass6 disposition block has an added function that assesses whether a thick film slide may be poorly prepared, based on the presence of very large numbers of distractor objects. If this condition is detected, the disposition block can adjust the diagnosis thresholds to reduce the chance that a malaria-negative but debris-filled slide will be diagnosed as malaria-positive.

\section{Synthesis block}

The thick and thin film algorithms are independent and can provide conflicting results for the same sample. The synthesis block combines the two findings as follows. It uses the thick film analysis to determine three results:

(i) Malaria diagnosis (i.e. whether malaria parasites are present). This matches standard microscopy practice [22].

(ii) Quantitation of parasites, at all parasitaemias. This differs from microscopy practice, which recommends switching to the thin film when the density on thick films is very high (roughly $80,000 \mathrm{p} / \mu \mathrm{L}$ ) [23] due to the difficulty of manually tracking large numbers of parasites in a single FoV. Because an automated system can readily track these higher counts, it can leverage the much larger blood volume on thick films to avoid Poisson variability in parasite counts (as provided in the supplementary information of reference [19]).

(iii) Determination of whether the species is P. falciparum, non-falciparum, or a mixed infection. This depends on the ratios between the numbers of latestage, $P$. falciparum ring-stage, and non-falciparum 
ring-stage parasites as well as the total ring-stage count. Mixed infections are identified by very high ring-stage counts (ascribed to P. falciparum, since non-falciparum species typically have lower parasitaemias) combined with sufficiently high late-stage counts (since $P$. falciparum typically presents very few late-stages).

The synthesis block only uses the thin film results if the thick film algorithm reports a non-falciparum or mixed species infection. Thin film results then determine the non-falciparum species, as in standard microscopy [22]. If the thick film result is non-falciparum or mixed species and the thin film result is negative, the species is predicted as $P$. vivax due to $P$. vivax being the most prevalent non-falciparum species of malaria globally [1].

The final report gives three parasitaemias: ring-stage (immature trophozoite), late-stage (mature trophozoite, schizont, and gametocyte), and total. It does not differentiate between the various late-stages. To evaluate quantitation accuracy on ECAMM P. falciparum slides, the ring-stage count was used rather than the total parasite count. This is because RITM's reference readings counted only asexual stages (i.e. non-gametocytes) consistent with malaria microscopy standards [22], and because non-gametocyte late-stages are uncommon in P. falciparum blood films.

The final report lists malaria species, WBC count, RBC count (thin film only), and poor slide quality warnings if indicated. It also includes a mosaic of highscoring objects' thumbnails for possible examination by the clinician.

\section{Results}

The EasyScan GO malaria detector correctly detected whether or not malaria was present in 33 of 35 slides (20 negative slides and 15 positive slides), for $94.3 \%$ accuracy. It achieved $82.9 \%$ accuracy for species ID, correctly labeling 29 of 35 slides. It correctly quantitated 10 of 20 slides, for $50 \%$ accuracy.

These results correspond to a Level 1, Level 2, and Level 1 microscopist for diagnosis, species ID, and quantitation, respectively, according to the rubric in [16] (shown in Table 1). A microscopist's final rating equals their lowest-scoring component, so the EasyScan GO would officially receive Level 2 microscopist status. These results are summarized in Table 2. The outputs from the malaria detector for each sample are given in Additional File 1. The results for each section are described in detail below.
Table 2 Performance of the EasyScan GO on the various components of the External Competence Assessment slide set

\begin{tabular}{lllll}
\hline $\begin{array}{l}\text { Component } \\
\text { of assessment }\end{array}$ & $\begin{array}{l}\text { Number } \\
\text { of slides in } \\
\text { subset }\end{array}$ & $\begin{array}{l}\text { Numbe } \\
\text { correct }\end{array}$ & $\begin{array}{l}\text { Percentage } \\
\text { correct }\end{array}$ & $\begin{array}{l}\text { WHO level } \\
\text { on this } \\
\text { component }\end{array}$ \\
\hline Detection & 35 & 33 & 94.3 & 1 \\
Species ID & 35 & 29 & 82.9 & 2 \\
Quantitation & 20 & 10 & 50 & 1 \\
\hline
\end{tabular}

\section{Parasite detection}

The malaria detector correctly identified whether or not malaria was present in 33 of 35 slides in the detection subset. All negative slides were classified as negative (100\% specificity), and 13 of 15 malaria positive slides were classified as positive ( $86.7 \%$ sensitivity). This yielded an accuracy of $94.3 \%$ (93.3\% balanced accuracy). The two false negative samples were $P$. falciparum samples with reference parasitaemias of $112 \mathrm{p} / \mu \mathrm{L}$ and $175 \mathrm{p} / \mu \mathrm{L}$. Also, all 20 P. falciparum quantitation slides were correctly classified as positive.

\section{Species ID}

The species was correctly labeled for 29 of the 35 slides (82.9\%) used for evaluating species identification accuracy. This included correctly labelling all 20 (100\%) negative samples, and 9 of 15 (60\%) positive samples, for a balanced accuracy of $80 \%$. Two errors were due to misclassifying 2 of the $7 P$. falciparum samples as negative. Also misclassified were 1 out of 3 Plasmodium malariae samples, both Plasmodium ovale samples, and the single mixed species ( $P$. falciparum plus $P$. ovale) sample. A confusion matrix is shown in Table 3 , which also gives (in parentheses) the performance on the 20 P. falciparum quantitation slides. Of the 20 quantitation slides, 19 were correctly classified as $P$. falciparum and one was incorrectly classified as Plasmodium vivax.

Performance on the simpler task of identifying $P . f a l$ ciparum versus non-falciparum was higher. When combining all $27 P$. falciparum-only samples in the 55-slide set, 24 were classified as $P$. falciparum, 1 was classified as $P$. vivax, and 2 were classified as negative $(88.9 \%$ accuracy). Of the 7 non-falciparum samples, 1 was classified as $P$. falciparum and the remaining 6 were classified as non-falciparum species $(85.7 \%$ accuracy). The mixed sample was classified as $P$. vivax.

\section{Quantitation}

Of the $20 \mathrm{P}$. falciparum slides used to evaluate quantitation, the EasyScan GO reported parasitaemias within $25 \%$ of the reference (manually counted) parasitaemias 
Table 3 Confusion matrix comparing the reported EasyScan GO species to the reference species. Values in parentheses are the results for slides not used for evaluating species identification accuracy

\begin{tabular}{|c|c|c|c|c|c|c|}
\hline \multirow[t]{2}{*}{ EasyScan GO species } & \multicolumn{6}{|c|}{ Reference species } \\
\hline & P. falc. & P. vivax & P. malariae & P. ovale & Negative & mixed $P f+P o$ \\
\hline P. falciparum & $5(19)$ & - & - & 1 & - & - \\
\hline P. vivax & $-(1)$ & 2 & 1 & - & - & 1 \\
\hline P. malariae & - & - & 2 & 1 & - & - \\
\hline P. ovale & - & - & - & 0 & - & - \\
\hline Negative & 2 & - & - & - & 20 & - \\
\hline
\end{tabular}

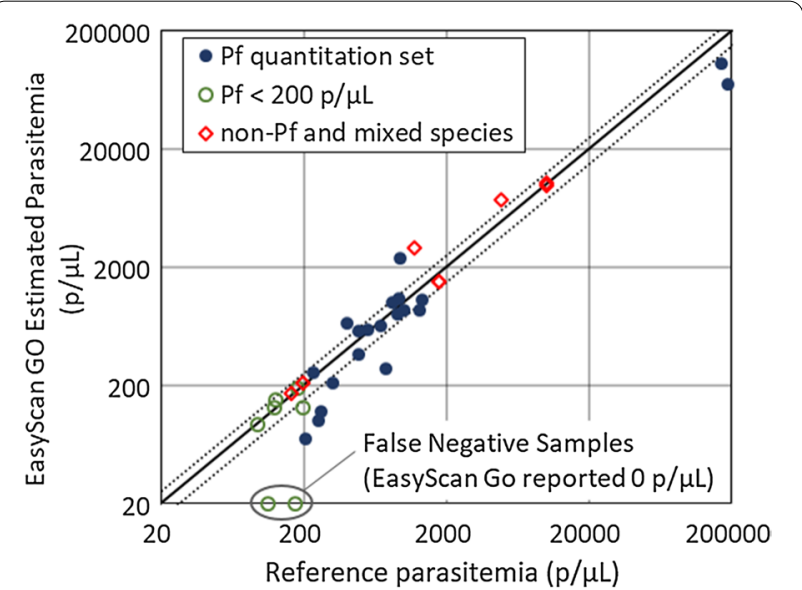

Fig. 3 Quantitation accuracy. EasyScan Go quantitation estimate vs. the reference quantitation. For P. falciparum samples, the ring-stage count is plotted, whereas for non-P. falciparum samples the total count is plotted. The dotted lines show $\pm 25 \%$ error

on 10 samples (50\%). Figure 3 plots the EasyScan GO parasitaemia vs. the reference parasitaemia for this set, as well as for the additional positive samples used for detection and species ID. Estimated parasitaemias were within $25 \%$ of reference parasitaemias for 4 of 7 (57.1\%) sub-200 $\mathrm{p} / \mu \mathrm{L} P$. falciparum samples, and 6 of 8 (75\%) non-falciparum or mixed species samples.

\section{Timing and invalid results \\ Timing}

The mean time to scan and analyse each slide in the configuration used for this assessment totalled 54.4 minutes: 12.8 and 5.8 minutes for scanning the thick and thin films respectively, and 28.7 and 7.0 minutes for analysing the thick and thin films, respectively. This is well above the 10 minutes per slide allowed in the WHO malaria microscopy quality assurance manual [16].

\section{Invalid results}

The system is designed to reject a film scan if there are not enough in-focus FoVs, too few WBCs (thick film), or too few RBCs (thin film). On all slides both thick and thin films were successfully scanned, with two exceptions. One thick film was rejected because every FoV was out-of-focus, but was re-scanned successfully. One thin film was rejected because all FoVs were reported as out-of-focus. Upon review, the entire thin film had a very dark background whether viewed with the EasyScan GO or with a standard manual microscope. An example FoV is shown in Additional File 2. Re-scanning was unsuccessful, because the malaria detector could not evaluate focus on the dark background. Since this sample's thick film was reported as $P$. falciparum, the lack of a thin film result did not affect its final disposition or the above analysis of accuracy.

\section{Discussion}

\section{WHO 55 as benchmark}

The WHO 55 is a template for slide sets used to evaluate malaria microscopist competence (ECAMM) as part of WHO's quality assurance program. Besides having a consistent composition, it assumes use of existing field infrastructure (i.e. field-prepared Giemsa-stained blood films), and emphasizes (in both content and scoring rubric) the key performance requirements of the most important use-cases. Thus, it may serve as a valuable benchmark dataset to assess and compare automated malaria detection systems. Such benchmarks are currently lacking.

In this paper, the performance of a fully-automated malaria diagnosis system, the EasyScan GO, on such a WHO 55 set is presented. The system scanned Giemsastained thick and thin films with an EasyScan GO automated microscope, then applied image-processing and ML methods to generate a slide-level diagnosis.

There are some limitations to using the WHO 55 template as a benchmark for testing automated systems. Each ECAMM slide set is a unique instance of the template, and performance on the set is only a single snapshot of system performance. The slide set is also fairly small, resulting in low statistical power. Ideally, a system should be tested on several ECAMM-style slide sets and should achieve Level 1 on most or all of them as confirmation of its performance. The ECAMM slide sets are also 
generally of high quality, and do not represent the high variability in sample preparation possible in the field. Internal evaluations (e.g. [5, 6]) and field trials (e.g. [7, 8], as well as ongoing studies) are also vital measures of system performance.

\section{Diagnosis}

The system achieved Level 1 performance on diagnosis of low-parasitemia slides, with $94.3 \%$ accuracy, $86.7 \%$ sensitivity, and $100 \%$ specificity. In the ECAMM slide set used, the non-falciparum slides had higher-than ideal parasitaemias. However, it has been observed (in prior experiments and field trials) that late-stage parasites of non-falciparum samples are much easier to detect and distinguish from distractor objects than are P. falciparum ring-stages, and the algorithm has consistently posted lower limits of detection for late-stages than for ringstages, making non-falciparum samples easier to diagnose at low parasitaemias than $P$. falciparum samples. It is, therefore, likely that the higher parasitaemias on nonfalciparum slides did not impact the system's sensitivity results.

\section{Quantitation}

The system also achieved Level 1 performance on quantitation of the 20 relevant $P$. falciparum samples, indicating its potential for use in new drug evaluation and drug resistance studies where accurate estimation of parasite densities is critical.

On the two high parasitaemia samples $(\sim 200 \mathrm{k} \mathrm{p} / \mu \mathrm{L})$, the system's estimates (done on thick film) were low relative to the reference counts (done on thin films [22]). Given a perfect detector, quantitations from thick films are intrinsically more consistent because the higher volume of blood reduces unavoidable Poisson variability. This is a case where machines have a distinct advantage over even expert humans because of their ability to screen many FoVs without fatigue and track large numbers of objects without error.

\section{Species ID}

The system achieved Level 2 performance on species ID. However, this score was likely inflated due to the differences in the ECAMM test set used compared to the ideal ECAMM template: there were fewer positive samples (15 vs. 20), the parasitaemia of samples was higher than 200 $\mathrm{p} / \mu \mathrm{L}$ for 6 (non-falciparum and mixed species) samples, and only one sample was mixed species instead of four. Had the test set matched the template, the system likely would have achieved Level 3 due to poor performance on mixed species samples and perhaps reduced performance on lower parasitaemia non-falciparum samples. The system's low species ID rating points to two weaknesses:

(i) Machine learning algorithms are often data-hungry [24], requiring far more training samples than humans do. The training sets were rich in $P$. falciparum and $P$. vivax, but contained few $P$. ovale and $P$. malariae samples since these are less common in malaria-infected humans. Plasmodium vivax training samples sufficed to ensure strong $P$. falciparum vs. non-falciparum classification on thick films, which enabled the system to have high accuracy at the level of $P$. falciparum vs. non-falciparum. But the vivax-ovale-malariae classifier suffered from lack of training data.

(ii) The system diagnoses mixed infections by leveraging priors on likely parasitaemias and ring-stage to late-stage ratios of the various species (non-falciparum samples tend to have lower maximum parasitaemias and $P$. falciparum late-stage counts tend to be low [25]). It has no way to distinguish mixed infections on low parasitaemia samples, because young ring-stage parasites look similar for all species. So in the absence of a distinctive P. falciparum gametocyte, any low-parasitaemia sample containing late-stages is classified as non-falciparum. Indeed, the ability of human microscopists to correctly classify such samples is a testament to their skill.

Fortunately, fine-grained species ID is a secondary need in some use-cases. One example is drug resistance studies which involve only quantitation of $P$. falciparum to generate parasite clearance curves. Additionally, geographic priors often indicate the likely type of nonfalciparum infection. Examples include: (a) In South America the two dominant species are $P$. vivax and $P$. falciparum [26], and Peruvian protocols allow use of thick films for species ID [27]; and (b) in West Africa, innate resistance to infection with $P$. vivax makes it rare compared to $P$. ovale [28]. The ECAMM template itself specifies incorporating "local prevalence" $[15,16]$. However, given travel and other considerations, these geographic priors are not certainties.

Species ID is important for some treatment decisions [29]. Examples include: (a) P. falciparum infection has a high fatality rate, so high $P$. falciparum sensitivity is important; and (b) P. vivax and P. ovale develop hypnozoites, which require distinct treatment, so accurate falciparum-malariae vs. vivax-ovale species ID is desirable. 


\section{Thick-film only evaluation}

The system mostly depends on thick film results, for various reasons:

(i) In many situations (e.g. drug resistance sentinel sites, and sites with only one non-falciparum species such as Peru), this is sufficient.

(ii) In the field, thin film quality can be highly variable (E. Long, pers. commun.), and thick film-only workflows can be preferable for various reasons ( $\mathrm{J}$. Carter, pers. commun.).

(iii) Machine learning algorithms are often data hungry, and owing to the greater density of blood on thick films the training sets contained more parasite images from thick films than from thin films.

However, successfully distinguishing between non-falciparum species using only thick films has not yet been achieved and the algorithm still depends on thin films for this task.

\section{ECAMM versions}

The "V2" ECAMM slide set [15] has some minor differences from "V1". These changes might have affected the system's performance, in either direction. Also, "V2" protocols specify that $30 \%$ of the slides be used during a preparation or orientation phase, i.e. the slide set is not treated as a true holdout set (a set of samples which is not seen prior to testing) since the microscopists receive some training on the slide set prior to assessment. Such prior exposure, if incorporated into training of an algorithm, would complicate the training flow and would also strongly affect machine results (by the nature of machine learning). In this regard, the "V2" protocols are not as well suited to evaluation of computer-automated systems.

\section{Timing}

Whether the current system runtime is acceptable depends on particular field constraints. In some field clinics, fast throughput is essential, and the system would be inappropriate for such scenarios. In other settings, the ability to analyse batches of samples overnight would mitigate long runtime (e.g. re-checking of slides for quality control).

Since these experiments were run, several changes have been made to the EasyScan GO software to shorten runtime, including:

(i) Scan a lower volume of blood, using thresholds on FoVs, WBCs (thick film) and RBCs (thin film), rather than scanning a fixed number of FoVs. This enables the blood volume scanned to more closely match manual microscopy protocols (100 FoVs with a $100 \times$ objective rather than the $\sim 580$ scanned here, and at least $500 \mathrm{WBCs}$ [22], rather than the average of $8000 \mathrm{WBCs}$ scanned here). Analysis time scales roughly linearly with the number of FoVs.

(ii) Analyze thick film first and proceed to thin film analysis only when indicated, i.e. only in case of a positive, non-falciparum result.

(iii) Perform scanning and analysis in parallel instead of in series, e.g. a second sample can be scanned while the first sample is being analysed.

Further experiments are needed to characterize the resulting changes in runtime, and to ensure there is no loss in accuracy due to the decreased scan area. Preliminary experiments indicate typical total time of $18-25$ minutes per slide with these changes. To simulate the effect on accuracy of reducing FoVs, the samples were reanalysed using 70 FoVs (the equivalent of $100 \mathrm{FoVs}$ using a standard microscope with a $100 \times$ objective), selected from the original scanned FoVs, for each thick film. This did not change the detection or species ID results for any sample, however the quantitation results varied by $0.7 \mathrm{x}$ to $2.3 \mathrm{x}$ compared to the original counts. A detailed discussion is beyond the scope of this report, but these results are included as columns in Additional file 1.

Finally, analysis time is dependent on computing power. This study was performed on a computer with specifications matching that provided as part of the EasyScan Go system - a relatively high-end laptop (as of 2019) with an Intel i7-9750H CPU, an NVIDIA GeForce GTX 1650 GPU, and 16 GB RAM.

\section{Machine vs. human trade-offs}

In malaria microscopy, as in other domains, machines have pros and cons relative to human workers:

(i) In general, machines can run long hours without getting tired, but conversely they are at risk of mechanical failures.

(ii) Once developed, machines can be deployed at scale, without personnel training bottlenecks (although for this application there remains a need for sample collection and preparation by trained personnel).

(iii) Machine learning algorithms are notoriously brittle in the face of test samples that differ markedly from their training data. In the malaria task, algorithm performance can degrade in deployments to new locations if the clinics' slide preparations have unfamiliar characteristics [7]. In such cases, humans 
adapt much more readily to the novel characteristics. For example, the thin film described in this report that was rejected by the system was, while highly abnormal, still interpretable by a human.

To develop ML algorithms for malaria is to enjoy ample opportunities to recognize and admire the skill, versatility, rapid learning, and adaptability of human workers. At the same time, EasyScan GO's strong performance on this benchmark slide set is evidence that fully-automated systems are poised to play a meaningful role in malaria field microscopy.

\section{Conclusions}

Automated (machine learning-based) systems are a promising way to improve the quality and consistency of malaria microscopy. The WHO 55 slide set, designed to evaluate microscopists' competence in crucial field usecases, can serve as a benchmark for evaluating such systems. The fully-automated EasyScan GO, a slide scanning microscope coupled with malaria detection algorithms, was evaluated on a WHO 55 slide set. It achieved Level 1 competence in Diagnosis and Quantitation, and Level 2 in Species ID, the best performance on this benchmark test of any fully-automated system to our knowledge. While runtime and species ID both require improvement for use in general case management settings, its strong results on this slide set indicate its potential value for research use-cases such as drug efficacy monitoring, and possibly for case management use-cases with less stringent species ID requirements.

\section{Supplementary Information}

The online version contains supplementary material available at https://doi. org/10.1186/s12936-021-03631-3.

Additional file 1:A full list of samples, reference results, and results of the malaria detector algorithms.

Additional file 2:An example Field of View from the unreadable thin film, compared to an acceptable FoV.

\begin{abstract}
Abbreviations
CNN: Convolutional neural network; ECAMM: External competence assessment of malaria microscopists; FoV: Field-of-view; ID: Identification; ML: Machinelearning; PCR: Polymerasechain reaction; RBC: Redblood cell; RITM: ResearchInstitute for Tropical Medicine; WBC: Whiteblood cell; WHO: WorldHealth Organization; WPRO: RegionalOffice for the Western Pacific.
\end{abstract}

\section{Acknowledgements}

The authors acknowledge the institutions that provided blood films for training the algorithm: Shoklo Malaria Research Unit, Amref Health Africa, University of Lagos, Universidad Peruana Cayetano Heredia, World Wide Antimalarial Resistance Network, Hospital for Tropical Diseases and the London School of Hygiene and Tropical Medicine, Kenya Medical Research Institute, Centers for Disease Control and Prevention, James Cook University, and the Defence
Services Medical Acadamy; and the Research Institute for Tropical Medicine for providing the ECAMM slide set.

\section{Authors' contributions}

MPH, CBD, LH, MSJ, CMT, SK, SJ, and CM developed the malaria detector algorithm. JL and CL provided the ECAMM slide set. SP, JYC, WO, DG, MD, RV, PLC, $B O, E G L, K T$, and TRB provided the microscope slides for training the algorithm. $\mathrm{TO}, \mathrm{MM}, \mathrm{RG}$, and $\mathrm{GY}$ annotated the training data and scanned the slides. DB and $\mathrm{KL}$ provided guidance in interpreting the test set and results, and CMB, $\mathrm{DB}, \mathrm{SP}, \mathrm{JC}, \mathrm{DG}, \mathrm{MD}, \mathrm{PLC}, \mathrm{EGL}$, and $\mathrm{KL}$ advised on malaria diagnosis use cases and needs. $\mathrm{MPH}, \mathrm{CBD}, \mathrm{CMB}$, and $\mathrm{CM}$ wrote, and all authors reviewed and approved, the manuscript. All authors read and approved the final manuscript.

\section{Funding}

Funding provided by The Global Good Fund I, LLC (www.globalgood.com). PLC is supported by the National Institute for Health Research Biomedical Research Centre, UCL Hospitals, London UK.

\section{Availability of data and materials}

The data supporting the conclusions of this article (results from the EasyScan Go) are included within the article and its additional file(s). Requests for access to ECAMM slide sets should be made to the WHO Collaborating Center for Malaria Diagnosis at RITM [18].

\section{Ethics approval and consent to participate}

Not applicable.

\section{Consent for publication}

Not applicable.

\section{Competing interests}

The authors declare that they have no competing interests.

\section{Author details}

1 Global Health Labs (formerly at Intellectual Ventures Laboratory/Global Good), 14360 SE Eastgate Way, Bellevue, WA 98007, USA. ${ }^{2}$ Applied Math Department, University of Washington, Seattle, WA 98195, USA. ${ }^{3}$ formerly Intellectual Ventures Laboratory, 3150 139th AVE SE, Bellevue, WA 98005, USA. ${ }^{4}$ Research Institute for Tropical Medicine, Muntinlupa, Philippines. ${ }^{5}$ Creative Creek LLC, Camano Island, WA, USA. ${ }^{6}$ Arizona State University, Tempe, AZ, USA. ${ }^{7}$ Independent Consultant, Issaquah, WA, USA. ${ }^{8}$ Shoklo Malaria Research Unit, Mahidol Oxford Tropical Medicine Research Unit, Faculty of Tropical Medicine, Mahidol University, Mae Sot, Thailand. ${ }^{9}$ Amref Health Africa, Nairobi, Kenya.

${ }^{10}$ University of Lagos, Lagos, Nigeria. ${ }^{11}$ Laboratorios de Investigacion y Desarrollo, Facultad de Ciencias y Filosofia, Universidad Peruana Cayetano Heredia, Lima, Peru. ${ }^{12}$ World Wide Antimalarial Resistance Network and MahidolOxford Tropical Medicine Research Unit, Bangkok, Thailand. ${ }^{13}$ Infectious Diseases Data Observatory and World Wide Antimalarial Resistance Network, Asia- Pacific Regional Centre, Bangkok, Thailand. ${ }^{14}$ Hospital for Tropical Diseases and the London School of Hygiene and Tropical Medicine, London, UK. ${ }^{15}$ Kenya Medical Research Institute, Nairobi, Kenya. ${ }^{16}$ Centers for Disease Control and Prevention, Atlanta, GA, USA. ${ }^{17}$ Defence Services Medical Academy, Mingaladon, Myanmar. ${ }^{18}$ Australian Institute of Tropical Health and Medicine, James Cook University, Cairns, Australia. ${ }^{19}$ Australian Defence Force Malaria and Infectious Disease Institute, Enoggera, Australia.

Received: 17 October 2020 Revised: 4 February 2021 Accepted: 6 February 2021

Published online: 25 February 2021

References

1. WHO. World Malaria Report 2019. Geneva, World Health Organization, 2019.

2. Zurovac D, Midia B, Ochola SA, English M, Snow RW. Microscopy and outpatient malaria case management among older children and adults in Kenya. Trop Med Int Health. 2006;11:432-40.

3. Nazare-Pembele G, Rojas L, Nunez FA. Lack of knowledge regarding the microscopic diagnosis of malaria by technicians of the laboratory network in Luanda, Angola. Biomedica. 2016;36:149-55. 
4. Poostchi M, Silamut K, Maude R, Jaeger S, Thoma G. Image analysis and machine learning for detecting malaria. Transl Res. 2018;194:36-55.

5. Delahunt CB, Jaiswal MS, Horning MP, Janko S, Thompson CM, Kulhare $S$, et al. Fully-automated patient-level malaria assessment on field-prepared thin blood film microscopy images. In: Proc Global Humanitarian Technologies Conf. 2019;1-8.

6. Mehanian C, Jaiswal M, Thompson C, Horning M, Ostbye T, McGuire S, et al. Computer-automated malaria diagnosis and quantitation using convolutional neural networks. In: IEEE Int Conf Comput Vis ICCV. 2017;116-25.

7. Torres K, Bachman CM, Delahunt CB, Alarcon BJ, Alava F, Gamboa VD, et al. Automated microscopy for routine malaria diagnosis: a field comparison on Giemsa-stained blood films in Peru. Malar J. 2018;17:339.

8. Vongpromek R, Proux S, Ekawati L, Archasuksan L, Bachman C, Bell D, Sutanto I, Dhorda M. Field evaluation of automated digital malaria microscopy: EasyScan GO. Trans R Soc Trop Med Hyg. 2019;113:14-5.

9. Yang F, Poostchi M, Yu H, Zhou Z, Silamut K, Yu J, Maude RJ, Jaeger S, Antani S. Deep learning for smartphone-based malaria parasite detection in thick blood smears. IEEE J Biomed Health Inform. 2020;24(5):1427-38.

10. Poostchi M, Ersoy I, McMenamin K, Gordon E, Palaniappan N, Pierce S, Maude RJ, Bansal A, Srinivasan P, Miller L, Palaniappan K, Thoma G, Jaeger S. Malaria parasite detection and cell counting for human and mouse using thin blood smear microscopy. J of Medical Imaging International Society for Optics Photonics. 2018;5(4):1-14.

11. Var E, Boray Tek F. Malaria parasite detection with deep transfer learning In: 3rd International Conference on Computer Science and Engineering (UBMK). 2018:298-302.

12. Masud M, Alhumyani $H$, Alshamrani SS, Cheikhrouhou O, Ibrahim S, Muhammad G, et al. Leveraging deep learning techniques for malaria parasite detection using mobile application. Wirel Commun Mob Comput. 2020;8895429.

13. Manescu P, Shaw MJ, Elmi M, Neary-Zajiczek L, Claveau R, Pawar V, et al. Expert-level automated malaria diagnosis on routine blood films with deep neural networks. Am J Hematol. 2020;95:883-91.

14. Chibuta S, Acar AC. Real-time malaria parasite screening in thick blood smears for low-resource setting. J Digit Imaging. 2020;33:763-75.

15. WHO. Malaria Microscopy Quality Assurance Manual V2. Geneva, World Health Organization, 2016.

16. WHO. Malaria Microscopy Quality Assurance Manual V1. Geneva, World Health Organization, 2009

17. Motic Digital Pathology. Xiamen, China. EasyScan GO Al-Powered Malaria Detection. https://moticdigitalpathology.com/EasyScanGo/. Accessed 1 February 2021.
18. WHO Collaborating Centre (WHOCC) for Malaria Diagnosis - Research Institute for Tropical Medicine. Republic of the Philippines. http://ritm. gov.ph/reference-laboratories/who-recognized-laboratories/who-colla borating-centre-whocc-for-malaria-diagnosis/. Accessed 14 October 2020.

19. Delahunt CB, Jaiswal MS, Horning MP, Janko S, Thompson CM, Kulhare S, et al. Fully-automated patient-level malaria assessment on field-prepared thin blood film microscopy images, including Supplementary Information. https://arxiv.org/abs/1908.01901. Accessed 1 February 2021.

20. Ren S, He K, Girshick R, Sun J. Faster R-CNN. Towards real-time object detection with Region Proposal Networks. IEEE Trans Pattern Anal Mach Intell. 2017;39:1137-49.

21. Chen T, Guestrin C. XGBoost. A scalable tree boosting system. In: Proc 22nd ACM SIGKDD. 2016;785 - 94

22. WHO. Basic Malaria Microscopy: Tutor's Guide. Geneva, World Health Organization, 2010

23. WHO. Malaria Microscopy Standard Operating Procedure MM-SOP-09: Malaria Parasite Counting. Geneva, World Health Organization, 2016.

24. Hestness J, Narang S, Ardalani N, Diamos G, Jun H, Kianinejad H, Patwary MA, Yang Y, Zhou Y. Deep learning scaling is predictable, empirically. https ://arxiv.org/abs/1712.00409. Accessed 1 February 2021.

25. WHO. Basic Malaria Microscopy: Learner's Guide. Geneva, World Health Organization, 2010

26. Recht J, Siqueira AM, Monteiro WM, Herrera SM, Herrera S, Lacerda MVG. Malaria in Brazil, Colombia, Peru and Venezuela: current challenges in malaria control and elimination. Malar J. 2017;16:273.

27. Ministerio de Salud, Peru. Manual de Procedimientos de Laboratoria Para el Diagnostico de Malaria. Lima, Ministerio de Salud, Peru, 2003.

28. Howes RE, Patil AP, Piel FB, Nyangiri OA, Kabaria CW, Gething PW, et al. The global distribution of the Duffy blood group. Nat Commun. 2011:2:266.

29. Centers for Disease Control and Prevention. CDC - Malaria - Diagnosis and Treatment (United States) - Treatment (U.S.) - Guidelines for Clinicians. https://www.cdc.gov/malaria/diagnosis_treatment/clinicians1.html. Accessed 1 February 2021.

\section{Publisher's note}

Springer Nature remains neutral with regard to jurisdictional claims in published maps and institutional affiliations.
Ready to submit your research? Choose BMC and benefit from:

- fast, convenient online submission

- thorough peer review by experienced researchers in your field

- rapid publication on acceptance

- support for research data, including large and complex data types

- gold Open Access which fosters wider collaboration and increased citations

- maximum visibility for your research: over $100 \mathrm{M}$ website views per year

At BMC, research is always in progress.

Learn more biomedcentral.com/submissions 